\title{
Propagation Characteristics of Electromagnetic Wave in Seawater Channel for Submerged Buoy
}

\author{
Yafeng Zhan, Xiaohan Pan \\ Tsinghua University, Beijing, China \\ Email: zhanyf@tsinghua.edu.cn
}

How to cite this paper: Zhan, Y.F. and Pan, X.H. (2019) Propagation Characteristics of Electromagnetic Wave in Seawater Channel for Submerged Buoy. Journal of Computer and Communications, 7, 72-81. https://doi.org/10.4236/jcc.2019.710007

Received: August 12, 2019 Accepted: October 9, 2019 Published: October 16, 2019

\begin{abstract}
The safety of submerged buoy is higher than traditional buoy. The most important problem for submerged buoy is that signal will be attenuated greatly due to ocean wave fluctuation and seawater. On the basis of ocean wave model, propagation characteristics of electromagnetic wave in seawater channel for submerged buoy is analyzed in this letter. It includes the propagation properties of electromagnetic wave in seawater and across the air-sea interface. The results show that the VHF frequency band, first order sea level and water depth of less than $10 \mathrm{~cm}$ are acceptable for submerged buoy.
\end{abstract}

\section{Keywords}

Submerged Buoy, Electromagnetic Wave, Seawater, Channel Characteristics

\section{Introduction}

Marine information construction is in the stage from Digital Ocean to Smart Ocean. As a result, the study of ocean sensors is blooming [1] [2], because it is the main method to forecast and monitor marine environment with advantages of all-weather, long-term and fixed-point [3]. Traditional buoys all float on the ocean surface, which is the fatal flaw for safety. Therefore, a submerged buoy is attractive because of long-term potential and higher security. It is already an important development direction of marine sensors [4].

Since most of the ocean buoys have a certain distance from the coast, and the space-based link can cover a wide range, the most effective way of real-time information transmission for ocean buoys is space-based link [5] [6] [7] [8]. Many existing literatures have investigated the propagation characteristics of traditional buoy communication channel [9] [10] [11], but the discussion about channel of submerged buoy is very limited. 
In this letter, we focus on the seawater channel characteristics for submerged buoy. First, the ocean wave model is given. Then, channel characterizations in seawater and across the air-sea interface are analyzed. Finally, the comprehensive simulation and analysis are presented.

\section{Ocean wave Modeling}

In actual environments, the ocean wave will fluctuate with the wind. The main way to describe ocean wave is wave spectrum $S(\omega, \theta)$ [12], and the wave amplitude $a$ is.

$$
a=\sqrt{2 S(\omega, \theta) \Delta \omega \Delta \theta}
$$

where $S(\omega, \theta)=S(\omega) D(\theta), S(\omega)$ is power spectrum, and $D(\theta)$ is direction spectrum [13].

$$
S(\omega)=\frac{\alpha g^{2}}{\omega^{5}} \exp \left[-\beta\left(\frac{g}{U \omega}\right)^{4}\right]
$$

where $\alpha=8.1 \times 10^{-3}, g$ is gravity acceleration, $\beta=0.74$ and $U$ is the wind speed above sea surface.

$$
D(\theta)=\frac{2}{\pi} \cos ^{2} \theta
$$

Theoretically, $\theta$ can range from $-\pi$ to $\pi$, but in reality most of ocean energy distributes in the range from $-\frac{\pi}{2}$ to $\frac{\pi}{2}$.

The simulation results of ocean wave with different wind speeds are shown in Figure 1. We can see the wave height increases with the increasing of wind speed.

\section{Propagation Properties in Sea Water}

Seawater is a conductive medium which has a great impact on the electromagnetic waves propagation. Because the Maxwell equations are the basic theory of the electromagnetic wave propagation, we use these equations to calculate and analyze the propagation properties of electromagnetic wave in seawater.

\subsection{Permittivity of Sea Water}

The permittivity of seawater can be calculated by an extension Debye formula called Havirial-Negami, which is expressed as [14],

$$
\varepsilon_{s w}=\varepsilon_{s w \infty}+\frac{\varepsilon_{s w 0}-\varepsilon_{s w \infty}}{1+\left(j 2 \pi f \tau_{s w}\right)^{1-\alpha}}-\frac{j \sigma_{s w}}{2 \pi f \varepsilon_{0}}
$$

where

$$
\begin{gathered}
\varepsilon_{s w 0}(T, S)=\varepsilon_{s w 0}(T) a(T, S), \\
\varepsilon_{s w \infty o}(T)=87.134-0.1949 T-0.01276 T^{2}+0.0002491 T^{3}, \\
a(T, S)=1+1.16 \times 10^{-5} T S-3.65 \times 10^{-3} S+3.21 \times 10^{-5} S^{2}-4.23 \times 10^{-7} S^{3},
\end{gathered}
$$




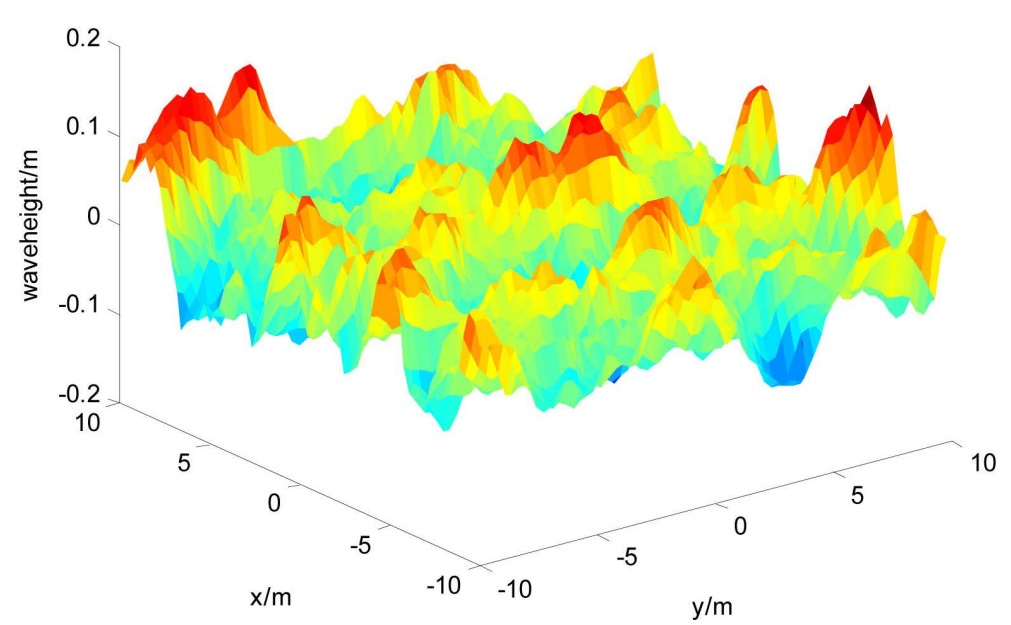

(a)

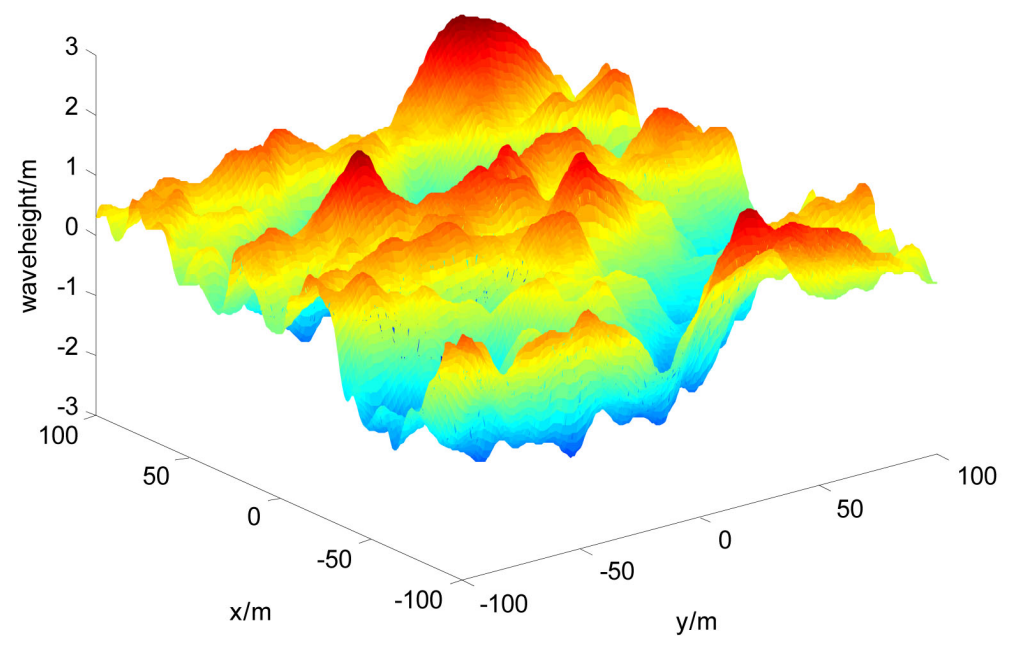

(b)

Figure 1. Wave simulation results with different wind speeds: (a) $3 \mathrm{~m} / \mathrm{s}$, (b) $11 \mathrm{~m} / \mathrm{s}$.

$$
\begin{gathered}
\tau_{s w}(S, T)=\tau(0, T) b(S, T), \\
\tau(0, T)=1.77 \times 10^{-11}-6.09 \times 10^{-13} T+1.10 \times 10^{-14} T^{2}-8.11 \times 10^{-17} T^{3}, \\
b(S, T)=1+2.28 \times 10^{-5} T S-7.64 \times 10^{-4} S-7.76 \times 10^{-6} S^{2}+1.11 \times 10^{-8} S^{3}, \\
\sigma_{s w}(S, T)=S\left(0.18-0.0015 S+2.09 \times 10^{-5} S^{2}-1.28 \times 10^{-7} S^{3}\right) \\
\quad \times \exp \left(( T - 2 5 ) \left(0.02+0.00013(25-T)+2.46 \times 10^{-6}(25-T)^{2}\right.\right. \\
\left.\left.-S\left(1.85 \times 10^{-5}-2.55 \times 10^{-7}(25-T)+2.55 \times 10^{-8}(25-T)^{2}\right)\right)\right)
\end{gathered}
$$

and $\varepsilon_{0}=8.854 \times 10^{-12}, \varepsilon_{\infty}=4.9, \alpha=0, S$ is salinity (\%o) and $T$ is temperature $\left({ }^{\circ} \mathrm{C}\right)$.

It is obvious that the permittivity of sea water has a relationship with frequency, temperature and salinity of seawater. We choose the most common case of seawater, a temperature of $15^{\circ}$ and a salinity of $35 \%$, as the simulation condition. The result is given in Figure 2. The permittivity becomes smaller as the frequency increases. 


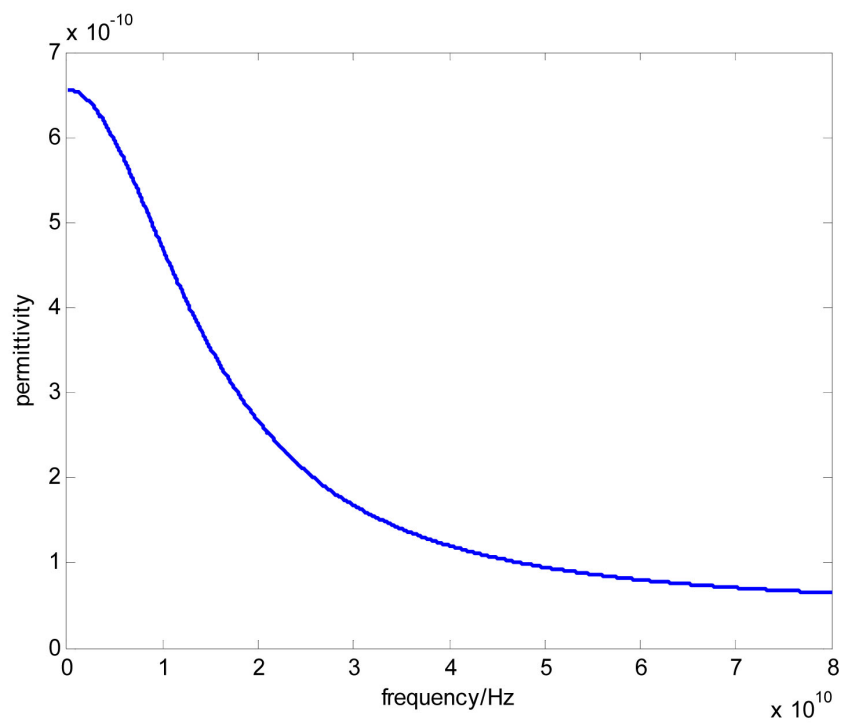

Figure 2. Permittivity of sea water for different frequencies with temperature $15^{\circ}$ and salinity $35 \%$.

\subsection{Channel Characterization}

Seawater is an electrical conductive medium where electromagnetic waves generate conduction current. Therefore, the Helmholtz equations of sea water are used.

$$
\begin{gathered}
\nabla^{2} E=\left(j \omega \mu \sigma-k^{2}\right) E=-\omega^{2} \mu \varepsilon\left(1-j \frac{\sigma}{\omega \varepsilon}\right) E=-\omega^{2} \mu \varepsilon_{e c} E \\
\nabla^{2} H=\left(j \omega \mu \sigma-k^{2}\right) H=-\omega^{2} \mu \varepsilon\left(1-j \frac{\sigma}{\omega \varepsilon}\right) H=-\omega^{2} \mu \varepsilon_{e c} H
\end{gathered}
$$

where $\mu=\mu_{0}$ is permeability and $\varepsilon_{e c}=\varepsilon[1-j \sigma / \omega \varepsilon]$ is the equivalent complex permittivity of seawater. With this parameter, we can consider seawater as an equivalent medium. The equivalent complex wave number is

$$
k_{c}^{\prime}=\omega \sqrt{\mu \varepsilon_{e c}}
$$

and the propagation constant is

$$
\gamma=j k_{c}^{\prime}=\alpha+j \beta
$$

where $\alpha$ is attenuation constant and $\beta$ is phase-shift constant. From (5) to (8), we can obtain

$$
\begin{aligned}
& \alpha=\omega \sqrt{\frac{\mu \varepsilon}{2}\left[\sqrt{1+\left(\frac{\sigma}{\omega \varepsilon}\right)^{2}}-1\right]} \\
& \beta=\omega \sqrt{\frac{\mu \varepsilon}{2}\left[\sqrt{1+\left(\frac{\sigma}{\omega \varepsilon}\right)^{2}}+1\right]}
\end{aligned}
$$

Substituting the above results into the plane wave expression, we can obtain,

$$
E=E_{0} e^{-\gamma z}=E_{0} e^{-\alpha z} e^{-j \beta z}
$$


where $\mathrm{z}$ is propagation distance. As can be seen in (11), the magnitude of the electric field strength decays exponentially with increasing propagation distance, and phase rotation occurs.

Some works have simulated the propagation of electromagnetic waves in seawater, but these works focus on the acoustic frequency band [15] [16]. Figure 3 illustrates the amplitude attenuation and phase shift caused by seawater in a wider frequency band.

From Figure 3 we can see that the VHF frequency band is the most suitable band due to its less attenuation and actual availability for space application. Therefore, in the following section, we simulate the properties of seawater channel in UHF band.

Furthermore, we simulate the amplitude attenuation with different depth in seawater. The result is shown in Figure 4. We can see that the attenuation is severe in seawater and gets worse with the increasing depth. So a depth of less than $10 \mathrm{~cm}$ should better be selected to ensure the communication quality.

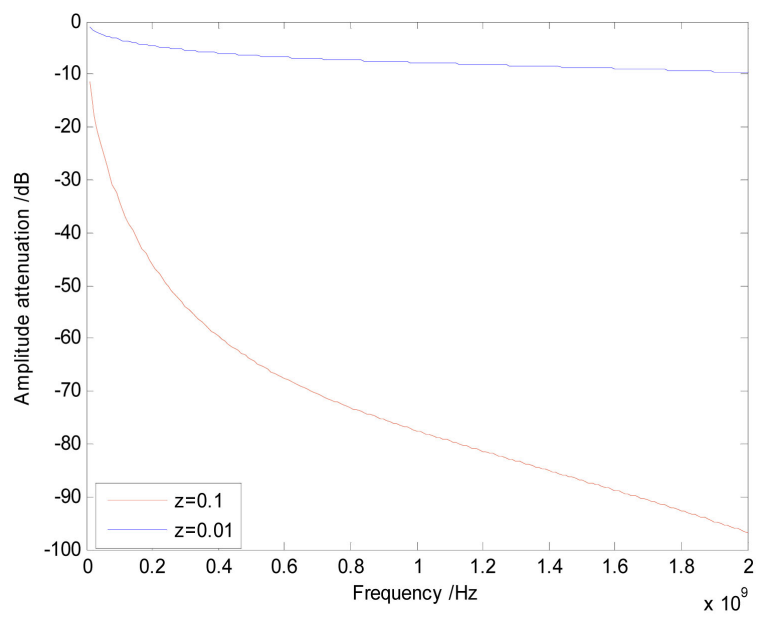

(a)

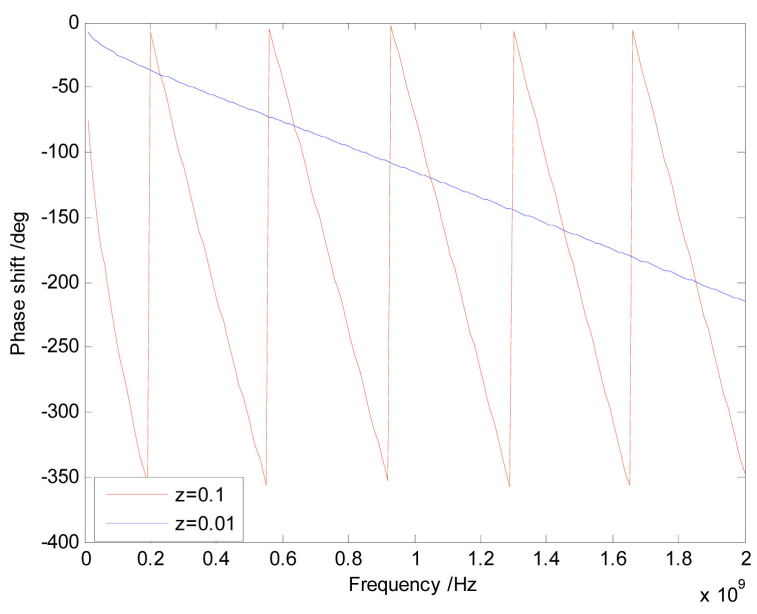

(b)

Figure 3. Amplitude attenuation and phase shift in seawater with different frequency. (a) Amplitude attenuation; (b) Phase shift. 


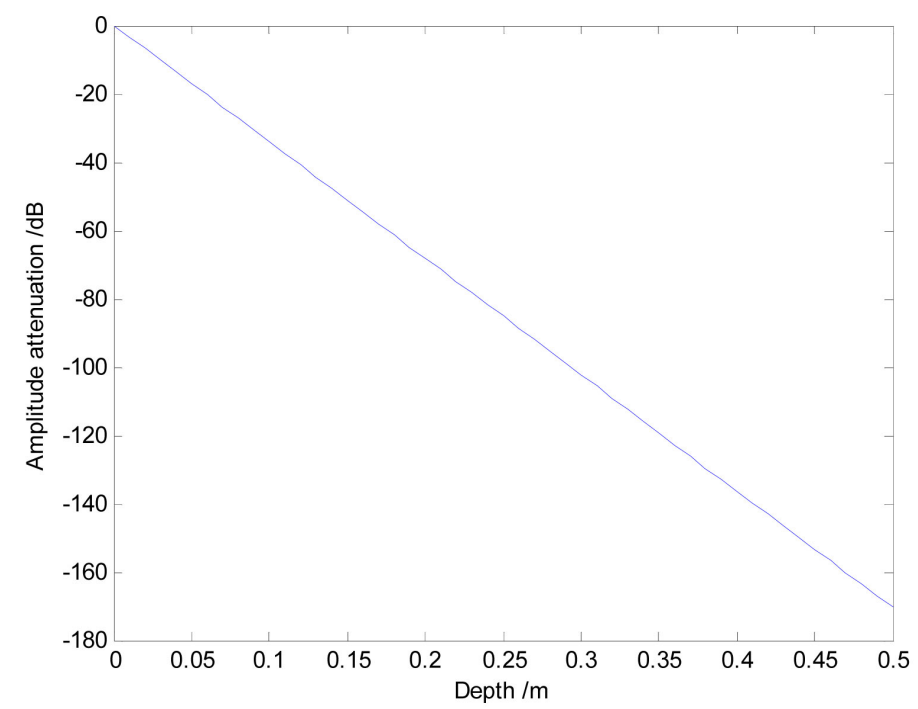

Figure 4. Amplitude attenuation in seawater with different depth and frequency 100 $\mathrm{MHz}$.

\section{Propagation Properties across the Air-Sea Interface}

For submerged buoy, electromagnetic waves are not only attenuated in seawater but also refracted through the air-sea interface. Since refraction will also produce attenuation, a study in propagation across air-sea interface is essential. The polarization mode of the incident wave field will have a great impact on the characteristics of transmitted wave field. Horizontal polarization and vertical polarization are two basic polarizations. Other polarization modes can be obtained by superposition of these two modes.

The refraction coefficient is defined as the ratio of amplitude of refracted wave to incident wave as the Fresnel formula shows [17].

$$
\begin{aligned}
T_{\perp} & =\frac{2 \eta_{2} \cos \theta_{1}}{\eta_{2} \cos \theta_{1}+\eta_{1} \cos \theta_{2}} \\
T_{\|} & =\frac{2 \eta_{2} \cos \theta_{1}}{\eta_{2} \cos \theta_{2}+\eta_{1} \cos \theta_{1}}
\end{aligned}
$$

where $\theta_{1}$ is the angle of incidence, $\theta_{2}$ is the angle of refraction which can be obtained from the Snell Law,

$$
\frac{\sin \theta_{1}}{\sin \theta_{2}}=\sqrt{\frac{\varepsilon_{2}}{\varepsilon_{1}}}
$$

where $\varepsilon_{1}$ is equal to the dielectric constant in freespace and $\varepsilon_{2}$ is the permittivity of seawater. $\eta_{1}$ and $\eta_{2}$ are intrinsic impedance which is the ratio of amplitude of the electric field to the magnetic field, and the equivalent complex intrinsic impedance $\eta_{c}$ can be expressed as

$$
\eta_{c}=\sqrt{\frac{\mu}{\varepsilon_{e c}}}=\sqrt{\frac{\mu}{\varepsilon-j\left(\frac{\sigma}{\omega}\right)}}
$$


Figure 5 illustrates the refraction coefficients of two kinds of polarization modes with different incidence angles. We can see that when incidence angle is zero, the refraction coefficients of these two modes are the same. The difference will appear when the incident is not perpendicular.

\section{Comprehensive Analysis and Simulation}

We simulated the total propagation characteristics of electromagnetic wave at a certain point, including the amplitude attenuation and phase shift both in seawater and across the ocean surface. Figure 6 gives the diagram of the propagation of electromagnetic waves in seawater channel. The simulation condition is the first order sea level which has a wind speed of $2.5 \mathrm{~m} / \mathrm{s}$, the frequency band is VHF, and the depth of submerged buoy is $10 \mathrm{~cm}$.

The simulation results are shown in Figure 7. It can be seen that in first order sea level, the seawater channel attenuation is about $40 \mathrm{~dB}$, which is rather applicable to the actual, while higher sea levels will generate more attenuation. This provides the basis for the design of communication systems.

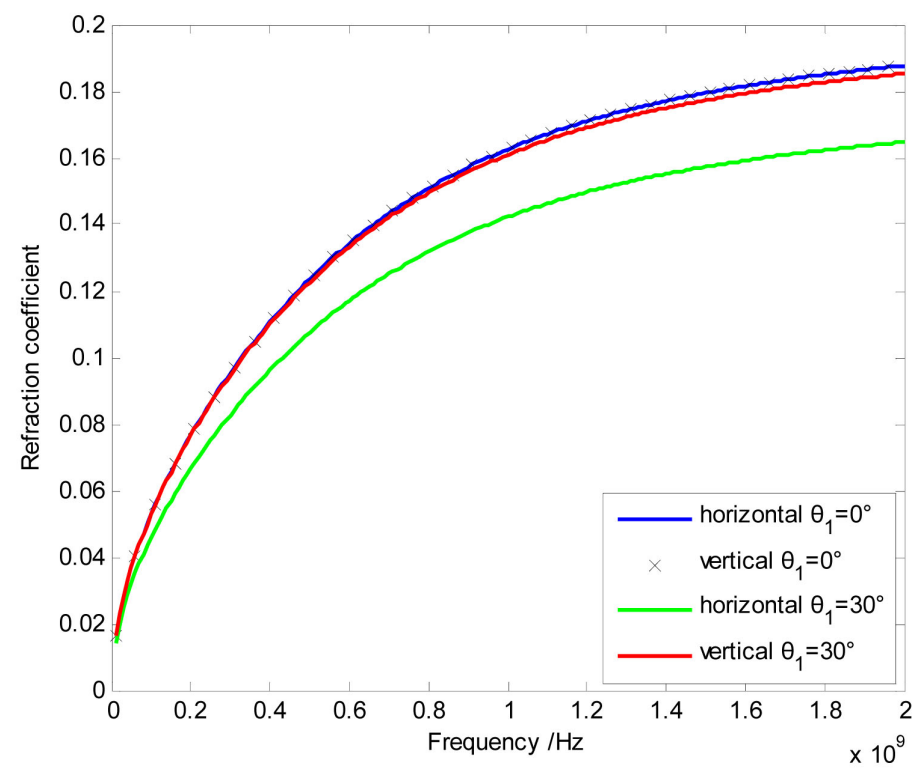

Figure 5. Refraction coefficients of two kinds of polarization modes with different incidence angles.

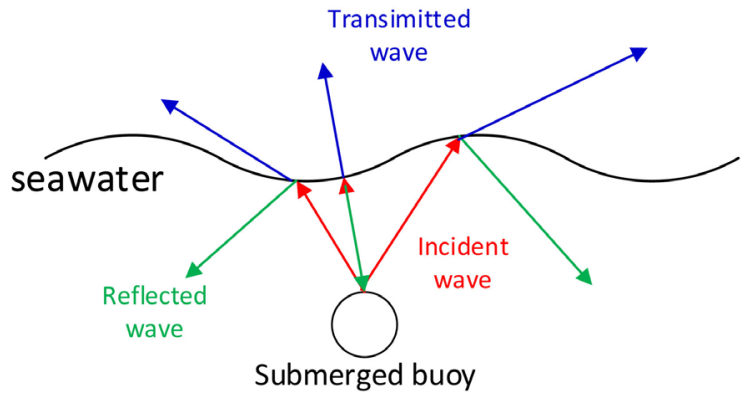

Figure 6. Diagram of the propagation of electromagnetic waves in seawater channel. 


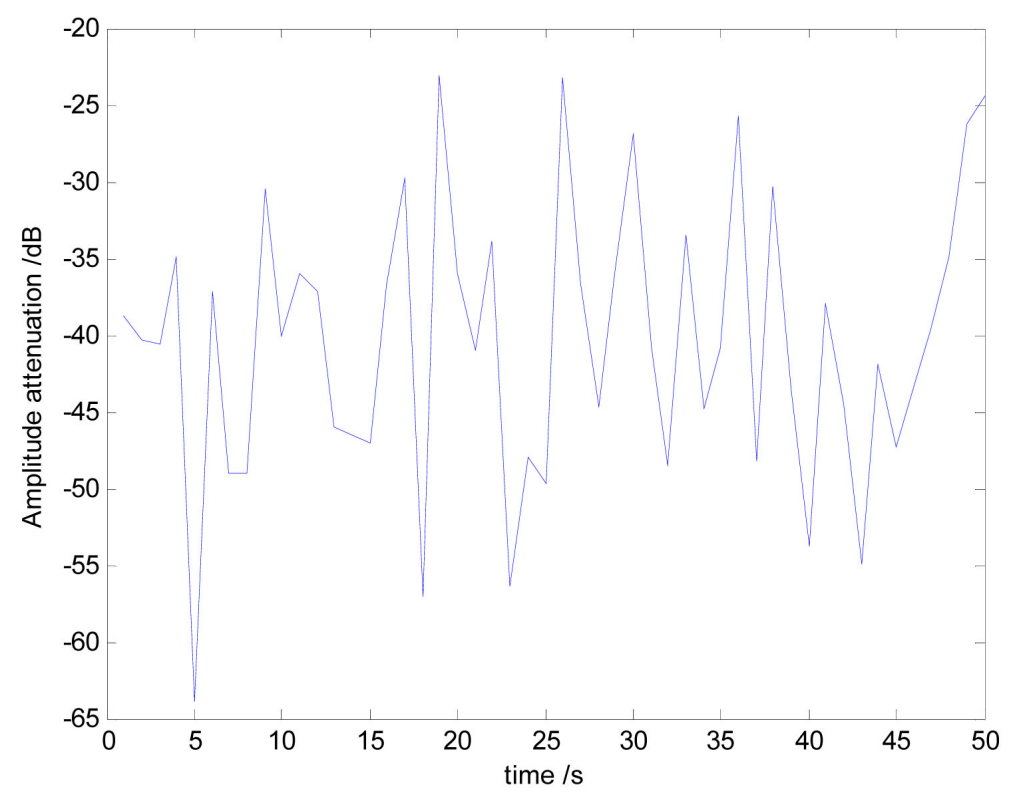

(a)

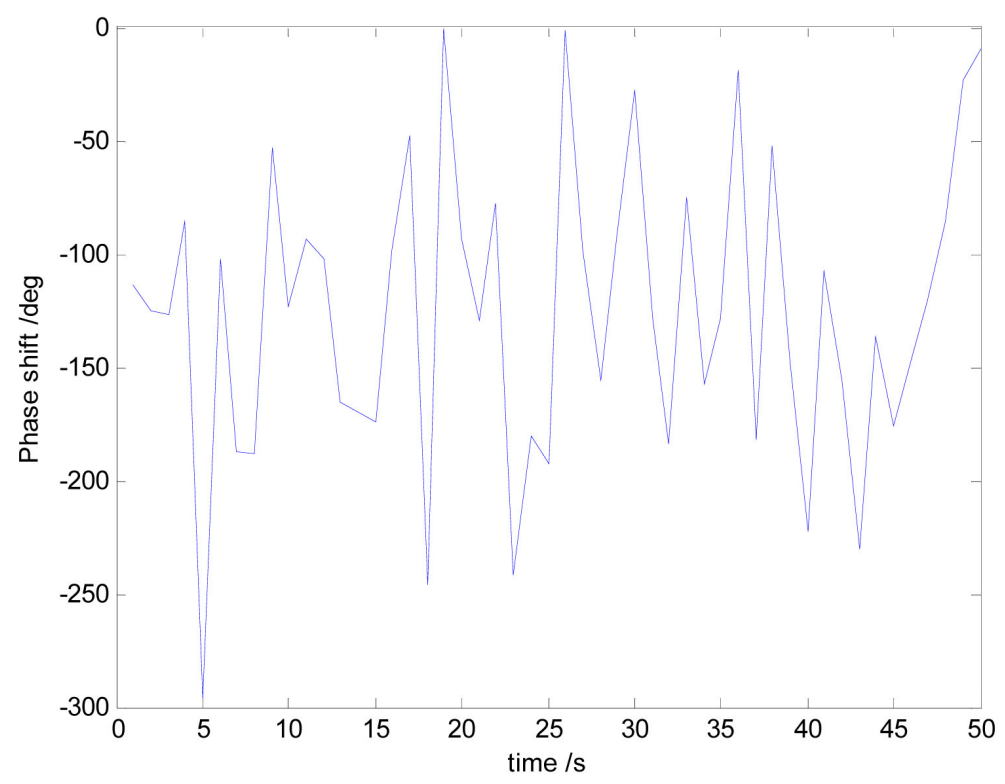

(b)

Figure 7. Comprehensive propagation characteristics in the condition of first order sea level. (a) Amplitude attenuation; (b) Phase shift.

\section{Conclusion}

The safety of traditional marine buoys is an important problem. In this regard, submerged buoy seems to have a better development prospect. However, many problems need to be overcome, such as a reliable information transmission. This letter analyzes the propagation characteristics of electromagnetic wave in seawater channel for submerged buoy. Amplitude variation and phase fluctuation of the communication signals are characterized. The results show that the VHF frequency band, first order sea level and water depth of less than $10 \mathrm{~cm}$ are ac- 
ceptable for submerged buoy. Such prior knowledge could help us to select a suitable algorithm for adaptive anti-rolling servo control and optimize waveform for resisting dynamic seawater attenuation.

\section{Acknowledgements}

This work was supported by the National Natural Science Foundation of China (61671263) and Tsinghua University Initiative Scientific Research Program (20161080057).

\section{Conflicts of Interest}

The authors declare no conflicts of interest regarding the publication of this paper.

\section{References}

[1] Toma, D.M., et al. (2011) Smart Sensors for Interoperable Smart Ocean Environment. Oceans, 19, 1-4.

[2] Cater, N.E. and Eng, P. (2009) Promoting Interoperable Ocean Sensors: The Smart Ocean Sensors Consortium. Oceans, 1-6.

[3] Zhang, J.Z. and Qin, J.C. (2012) Simulation and Design of Buoy Communication. Radio Communications Technology.

[4] Raimondi, F.M., et al. (2015) An Innovative Monitoring Underwater Buoy Systems (MUnBuS) for Marine and Rivers installation with IR-Cam, Instrumental Telemetry and Acoustic Data Acquisition Capability. Oceans, 1-7. https://doi.org/10.1109/oceans-genova.2015.7271600

[5] Myers, R.G., et al. (2008) A New Tsunami Detection Concept Using Space-based Microwave Radiometery. Geoscience and Remote Sensing Symposium. https://doi.org/10.1109/igarss.2008.4779883

[6] Stephen, C.R., et al. (2016) Fifteen Years of Ocean Observations with the Global Argo Array. Nature-Climate Change, 6, 145-153.

[7] Shaumeyer, J.N. and Borden, J.M. (2000) An Advanced Buoy with High-Volume, Two-Way LEO Satellite Communications. Oceans 2000 MTS/IEEE conference and Exhibition, 1103-1108. https://doi.org/10.1109/oceans.2000.881750

[8] O'reilly, T., et al. (2007) Satellite Link Management for an Ocean Observation Network. Oceans, 1-9.

[9] Lee, Y.H., Dong, F. and Meng, Y.S. (2014) Near Sea-Surface Mobile Radiowave Propagation at $5 \mathrm{GHz}$ : Measurements and Modeling. Radioengineering, 23, 824-830.

[10] Macmillan, A., Marina, M.K. and Triana, J.T. (2010) Slow Frequency Hopping for Mitigating Tidal Fading on Rural Long Distance Over-Water Wireless Links. INFOCOM IEEE Conference on Computer Communications Workshops, 1-5. https://doi.org/10.1109/infcomw.2010.5466612

[11] Zhang, W., Bai, S., Liu, Y., et al. (2010) Cognitive Radio Scheduling for Overwater Communications. Global Telecommunications Conference (GLOBECOM), 1-5. https://doi.org/10.1109/glocom.2010.5683565

[12] Amouzegar, M. (1999) A Global Optimization Method for Nonlinear Bilevel Programming Problems. IEEE Trans. on System, Man, Cybernetics-Part B, 29, 771-776. 
https://doi.org/10.1109/3477.809031

[13] Li, C.Z. (2009) Atmosphere/Ocean Interface Channel Research of Laser Communication. Huazhong University of Science \& Technology.

[14] Debye, P. (1929) Polar Molecules. Reinhold, New York.

[15] Zhu, H.-R., Zhang, B.-T. and Chen, B. (2013) Propagation Characteristics of U1tra-Low Frequency Electromagnetic Wave in Seawater. Command Control \& Simulation, 35.

[16] Chen, C., Zhou, J. and Gong, S.-G. (2004) Propagation Properties of Electromagnetic Wave in Sea Water. Journal of Naval University of Engineering, 16.

[17] Zhou, X.L. (2008) Electromagnetic Field. Publishing House of Electronics Industry, 163-167. 